\title{
Dysembryoplastic Neuroepithelial Tumor: A Rare Brain Tumor with Excellent Seizure Control after Surgical Resection
}

\author{
Rajnish Kumar Arora ${ }^{1}$ Pranshu Bhargava ${ }^{1}$ Poonam Arora ${ }^{2}$ Prashant Joshi ${ }^{3} \quad$ Garga Basu ${ }^{1}$ \\ Radhey Shyam Mittal ${ }^{1}$ \\ 1Department of Neurosurgery, All India Institute of Medical \\ Sciences, Rishikesh, Uttarakhand, India \\ ${ }^{2}$ Department of Anesthesiology, All India Institute of Medical \\ Sciences, Rishikesh, Uttarakhand, India \\ ${ }^{3}$ Department of Pathology, All India Institute of Medical Sciences, \\ Rishikesh, Uttarakhand, India \\ Address for correspondence Pranshu Bhargava, MS MCh (Neurosurgery), \\ Department of Neurosurgery, All India Institute of \\ Medical Sciences, Rishikesh, Uttarakhand 249203, India \\ (e-mail: bhargava_pranshu@rediffmail.com). \\ Indian J Neurosurg 2022;11:86-92
}

\begin{abstract}
Introduction Dysembryoplastic neuroepithelial tumor (DNET) is a rare cause of intractable epilepsy, which has excellent results in terms of seizure control after surgical resection. We present one such case, because of its rarity, to highlight the effect of tumor removal on seizure control, particularly DNET.

Materials and Methods/Case Summary A 9-year-old male patient presented with sudden onset of partial seizures for the past 6 months. There were five episodes. In each episode, the patient engaged in irrelevant talk, followed by deviation of mouth to left and twitching movements. The episode lasted 5 minutes and there was no loss of consciousness. There was no aura or tongue bite, and in one of the episodes, the patient lost consciousness. There was no other significant positive history. On examination, the child was consciously alert, without focal neurological deficit or features of meningitis. There was no papilledema. The patient was on phenytoin sodium, phenobarbitone and clobazam. Magnetic resonance imaging (MRI) of brain was done with and without contrast. MRI revealed a lesion approximately $4.1 \times 3.6 \times 3.2 \mathrm{~cm}$ in the right medial temporal lobe. It was hypointense to brain on $\mathrm{T} 1$ and fluid-attenuated inversion recovery (FLAIR), and hyperintense on T2-weighted images. Diffusion restriction was present and there was minimal contrast uptake. There was no evidence of mass effect or midline shift After discussing the risks and benefits with parents, the patient underwent preanesthetic checkup, and was taken up for craniotomy and excision of tumor. Gross total excision was done. The child was started orally on day 1 postoperatively and ambulated. There were no further seizure episodes. The

Keywords

- dysembryoplastic

- neuroepithelial

- seizure patient was continued on phenytoin and clobazam, and phenobarbitone was tapered gradually. At 6 months, the child was seizure-free.

Conclusions DNET are rare tumors occurring early in life and presenting with intractable seizures. Surgical resection offers a good and safe chance for long-term seizure control.
\end{abstract}

DOI https://doi.org/ $10.1055 / \mathrm{s}-0040-1713561$ ISSN 2277-954X. (c) 2021. Neurological Surgeons' Society of India.

This is an open access article published by Thieme under the terms of the Creative Commons Attribution-NonDerivative-NonCommercial-License, permitting copying and reproduction so long as the original work is given appropriate credit. Contents may not be used for commercial purposes, or adapted, remixed, transformed or built upon. (https://creativecommons.org/licenses/by-nc-nd/4.0/).

Thieme Medical and Scientific Publishers Pvt. Ltd. A-12, 2nd Floor, Sector 2, Noida-201301 UP, India 


\section{Introduction}

The term dysembryoplastic neuroepithelial tumor (DNET) was first described in 1988 by Dauport et $\mathrm{al}^{1}$ to describe a unique histological pattern of brain tumors associated with medically intractable partial seizures which are surgically curable. Recent studies have found surgical resection to be effective in seizure control in $98 \%$ of patients. ${ }^{2}$ We report a 9-year-old male child presenting with recurrent partial seizures over the past 6 months, which is uncontrolled on polytherapy. Complete resection was done, following which patient was seizure-free at 6 months. Histopathology revealed the tumor to be DNET.

This case is reported because of its rarity and ability to highlight the effect of tumor removal on seizure control, particularly DNET.

\section{Case Summary}

A 9-year-old male patient presented with sudden onset of partial seizures for the past 6 months. The patient experienced a total of five episodes. During each episode, the patient engaged in irrelevant talk followed by deviation of mouth to left and twitching movements. The episode lasted 5 minutes and there was no loss of consciousness. There was no aura or tongue bite, but in the last episode, the patient lost consciousness.

There was no history of head trauma, high fever, vomiting, headache, and visual or auditory symptoms. No history suggestive of motor weakness or sensory loss, cranial nerve involvement, as well as no bladder or bowel complaints. The child was delivered normally at full term and cried after birth, so no history to suggest birth asphyxia or neonatal infection. Developmental milestones were normal. The child recorded average scholastic performance.

On examination, the child was found to be consciously alert and interactive. There was no focal neurological deficit or features of meningitis. No neurocutaneous markers were seen. There was no papilledema.

The patient was started sequentially on phenytoin sodium, phenobarbitone, and clobazam on weight-adjusted doses. However, seizures were still not controlled at 6 months.

Magnetic Resonance Imaging (MRI) of brain was done with and without contrast. MRI revealed a lesion approximately $4.1 \times 3.6 \times 3.2 \mathrm{~cm}$ in the right medial temporal lobe. It was hypointense to brain on T1 ( - Fig. 1) and fluid-attenuated inversion recovery (FLAIR), hyperintense on T2-weighted images (-Fig. 2). Diffusion restriction was present and there was minimal contrast uptake (-Fig. 1). There was no evidence of mass effect or midline shift.

After discussing the risks and benefits with parents, the patient underwent preanesthetic checkup and was also taken up for craniotomy and excision of tumor. A right frontotemporal craniotomy was done in the supine position. Under microscopic view, gross total excision was done. The tumor was located in the right temporal lobe posteriomedially. It was greyish soft, moderately vascular, and suckable except at the periphery. After assuring hemostasis dura was primarily closed and bone flap was replaced. The child was reversed and intubated. There were no intra or postoperative complications or seizure episodes.

Postoperative computerized tomogram (CT) showed adequate decompression and no hematoma formation ( - Fig. 3).

The child was started orally on day 1 postoperatively and ambulated. There were no further seizure episodes.

The patient was continued on phenytoin and clobazam, and phenobarbitone was tapered gradually.

MRI at 3 months revealed no recurrence or residual tumor (-Fig. 4, 5). At 6 months, the child was doing well with no cognitive impairments. It was planned to stop clobazam at the 1-year mark. The patient's parents were advised follow-up every 3 months.

Histopathology on routine hematoxylin and eosin revealed multiple glial tissue bits, showing a cellular tumor

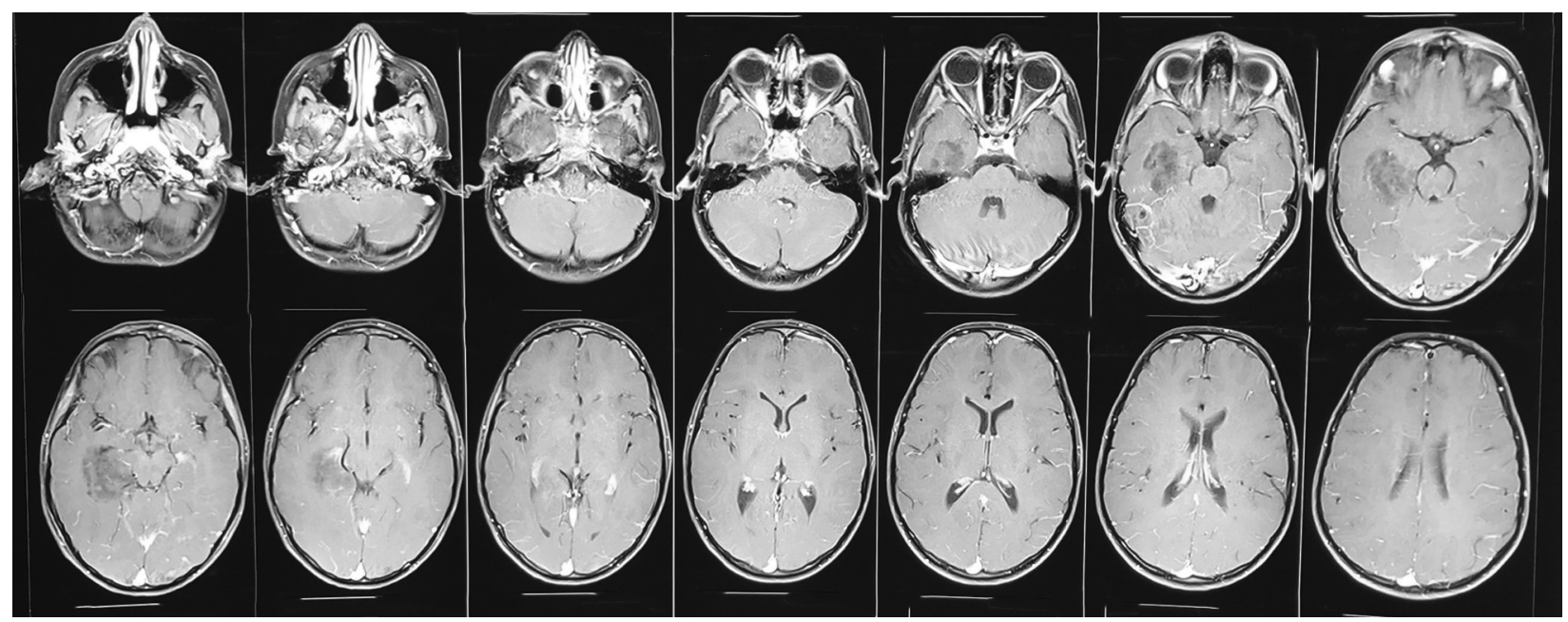

Fig. 1 Preoperative T1 contrast magnetic resonance resonance imaging. 


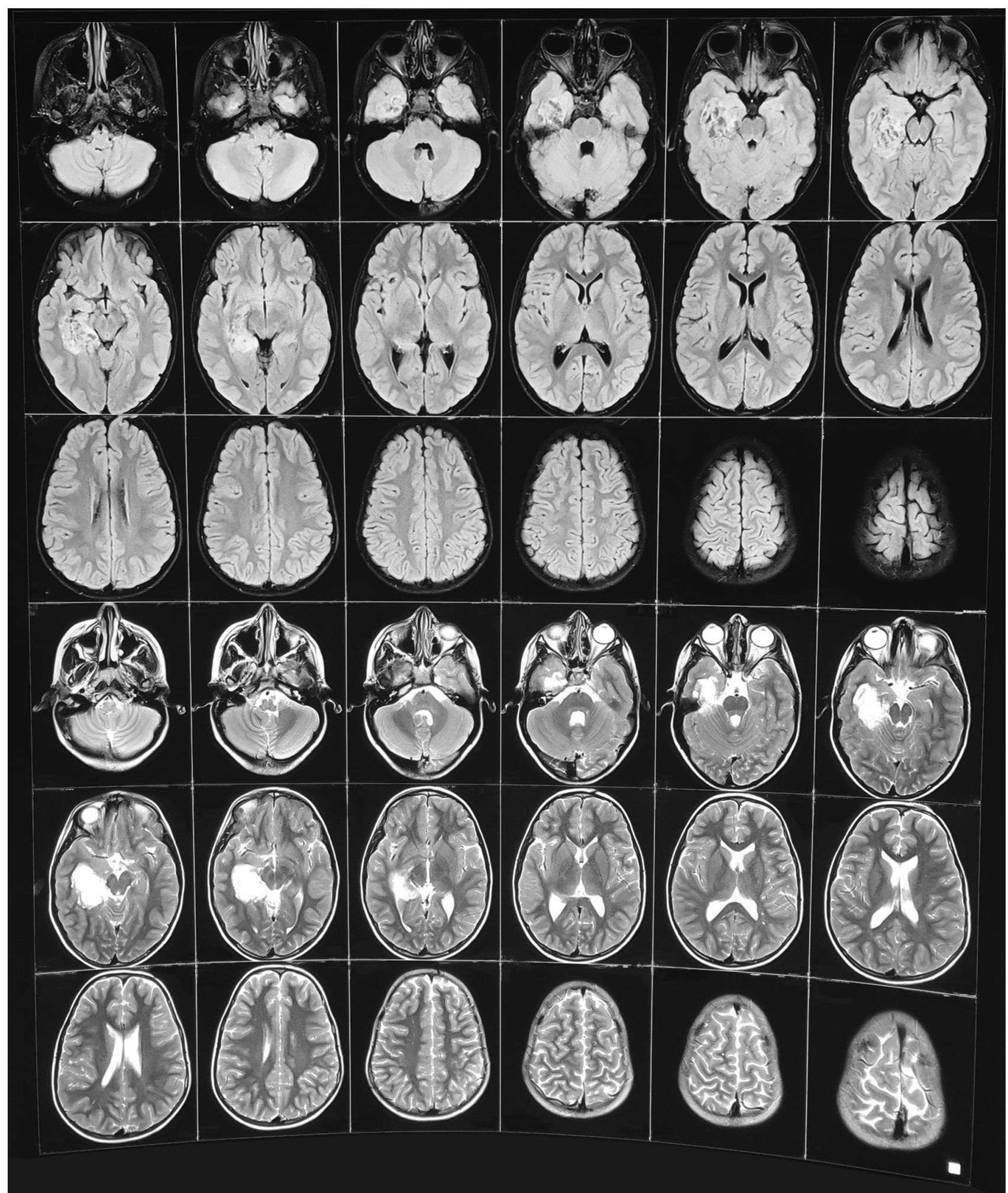

Fig. 2 Preoperative T2 and fluid-attenuated inversion recovery magnetic resonance resonance imaging.

arranged in multinodular pattern and composed of round to oval oligodendrocyte-like cells and axons (-Fig. $6 \mathbf{6 A}$ ). Interspersed were normal glial tissue and floating neurons (-Fig. 6C). There were focal areas of mucoid-like matrix and microcystic change ( - Fig. 6B). Focal cortical dysplasia was also seen. On immunohistochemistry, isocitrate dehydrogenase (IDH) was negative, glial fibrillary acidic protein (GFAP) was noncontributory, synaptophysin and chromogranin was positive, and Ki67 labeling index was less than $1 \%$. Cells were positive for Neuronal Nuclei $($ NeuN) ( - Fig. 6D). These features were considered consistent with a pathological diagnosis of DNET. 


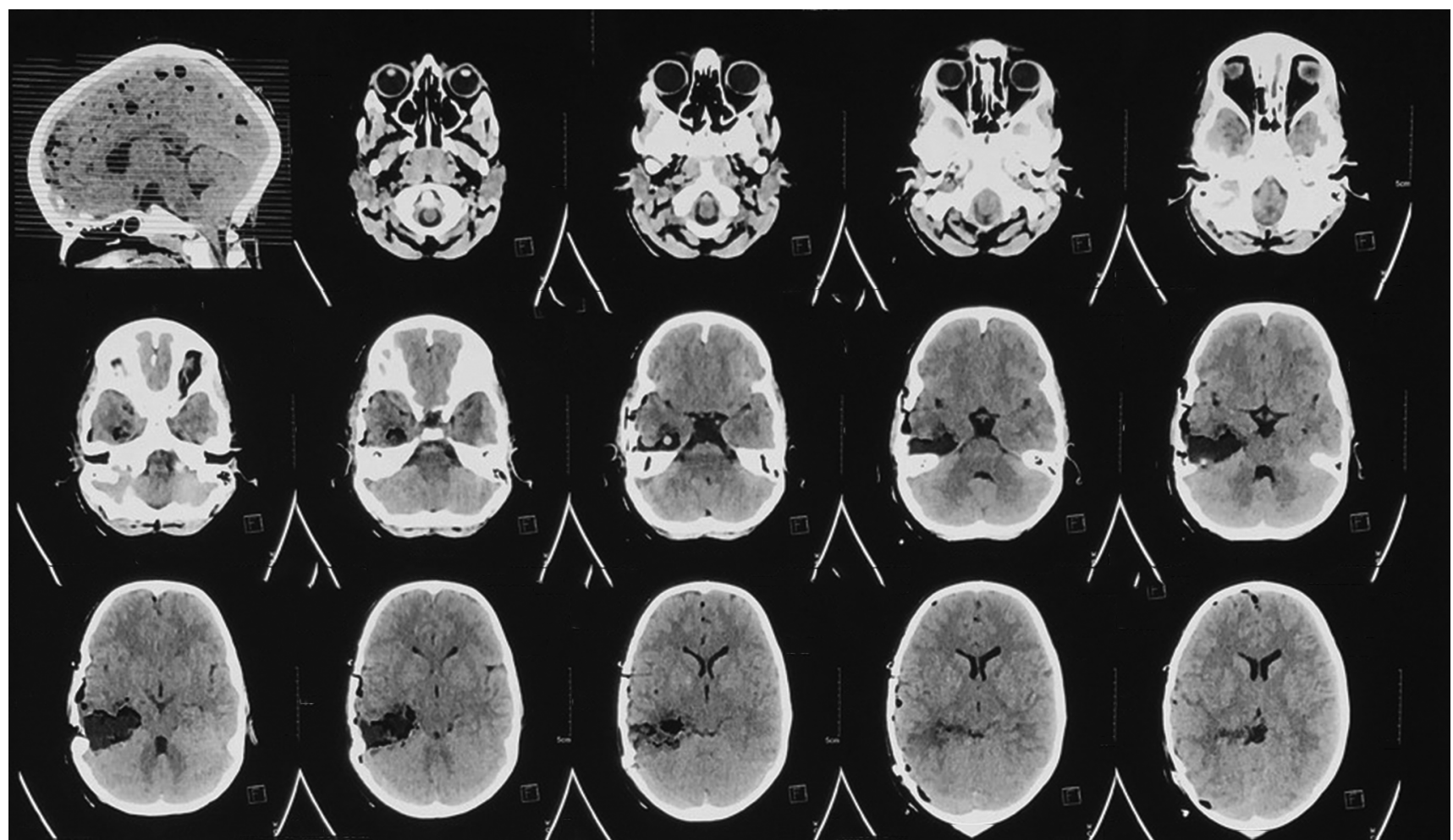

Fig. 3 Immediate postoperative computerized tomography scan.

\section{Discussion}

DNET as a distinct pathological entity has been welldocumented after its description by Dauport et al. ${ }^{1}$ They are usually seen in young adults, with the most common site of occurrence being the temporal lobes, but they may also occur at other sites. ${ }^{1-4}$

Common clinical features include a history of partial seizures, with or without generalization, onset before 20 years of age, and absence of neurologic deficit ${ }^{3}$ Our patient's profile is consistent with these clinical criteria.

MRI is the investigation of choice. Commonly seen features are cortical location of the lesion, cystic appearance, absence of mass effect or peritumoral edema. Other features are calcification, absence of contrast enhancement, and scalloping of the overlying cortical bone Differential diagnosis include lowgrade glioma and ganglioglioma. FLAIR and apparent diffusion coefficient (ADC) may be helpful in distinguishing them.,4

Surgical resection of tumor is documented to be effective in long-term seizure control in cases of DNET. ${ }^{1,2}$ It has been also found that it is safe in terms of mortality as well as morbidity. Lesionectomy, with or without additional resection, and use of brain mapping are the available treatment options. ${ }^{2}$ Recurrence is rare. In a review by Ranger and Diosy, they found surgical resection effective in improving control in $98 \%$ of patients and long-term seizure freedom in $86 \%$. They found no deaths (immediate or delayed). Recurrence was seen in two out of 185 patients Hence, it is of importance to recognize this rare entity where excellent seizure control is possible with minimal morbidity and mortality. ${ }^{2}$ In our patient, we were able to stop one of the three drugs (phenobarbitone) and planned to stop clobazam subsequently.

\section{Conclusion}

DNET are rare tumors occurring early in life, presenting with intractable seizures. Surgical resection offers a good and safe chance for long-term seizure control. 


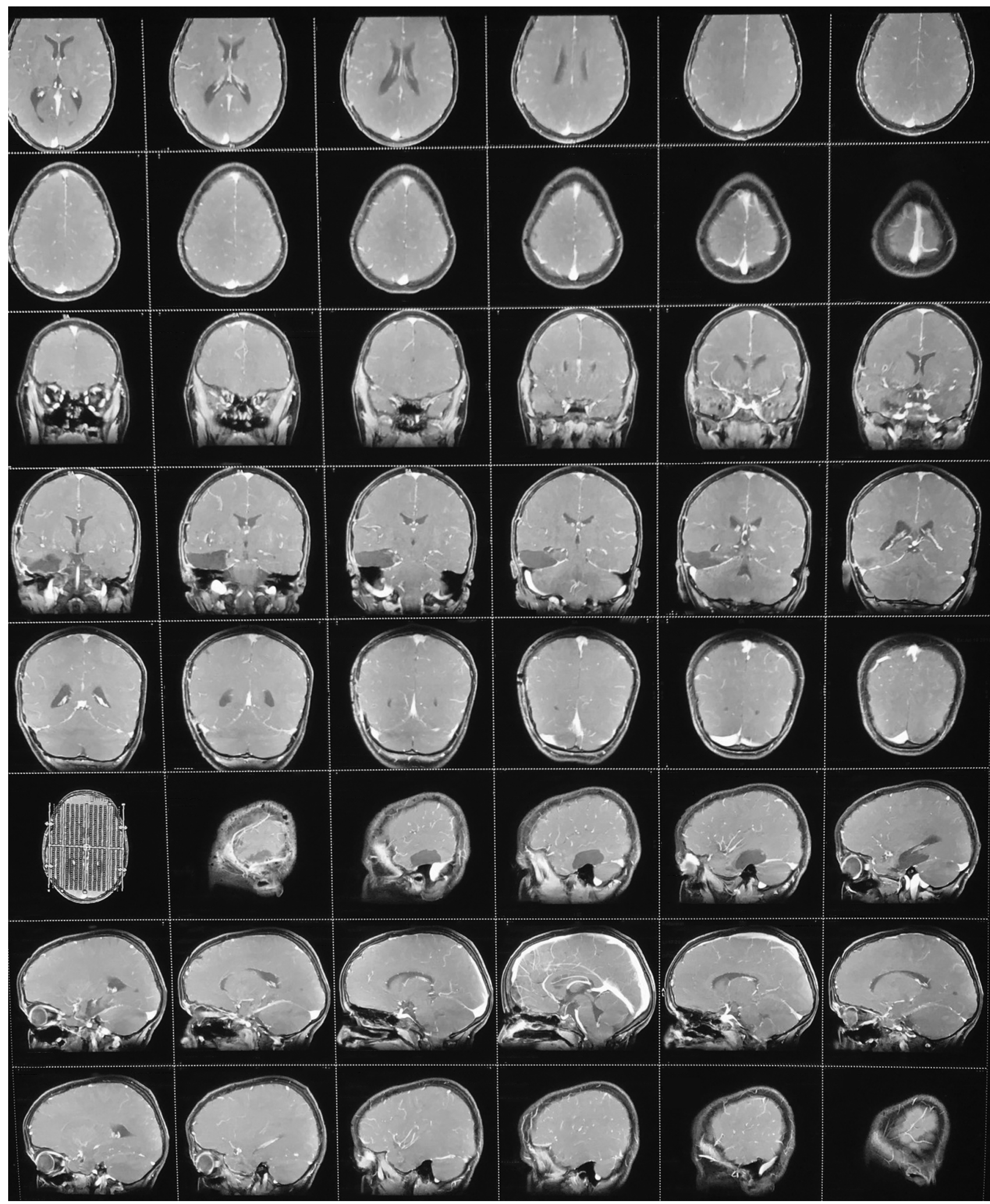

Fig. 4 Postoperative T1 contrast. 


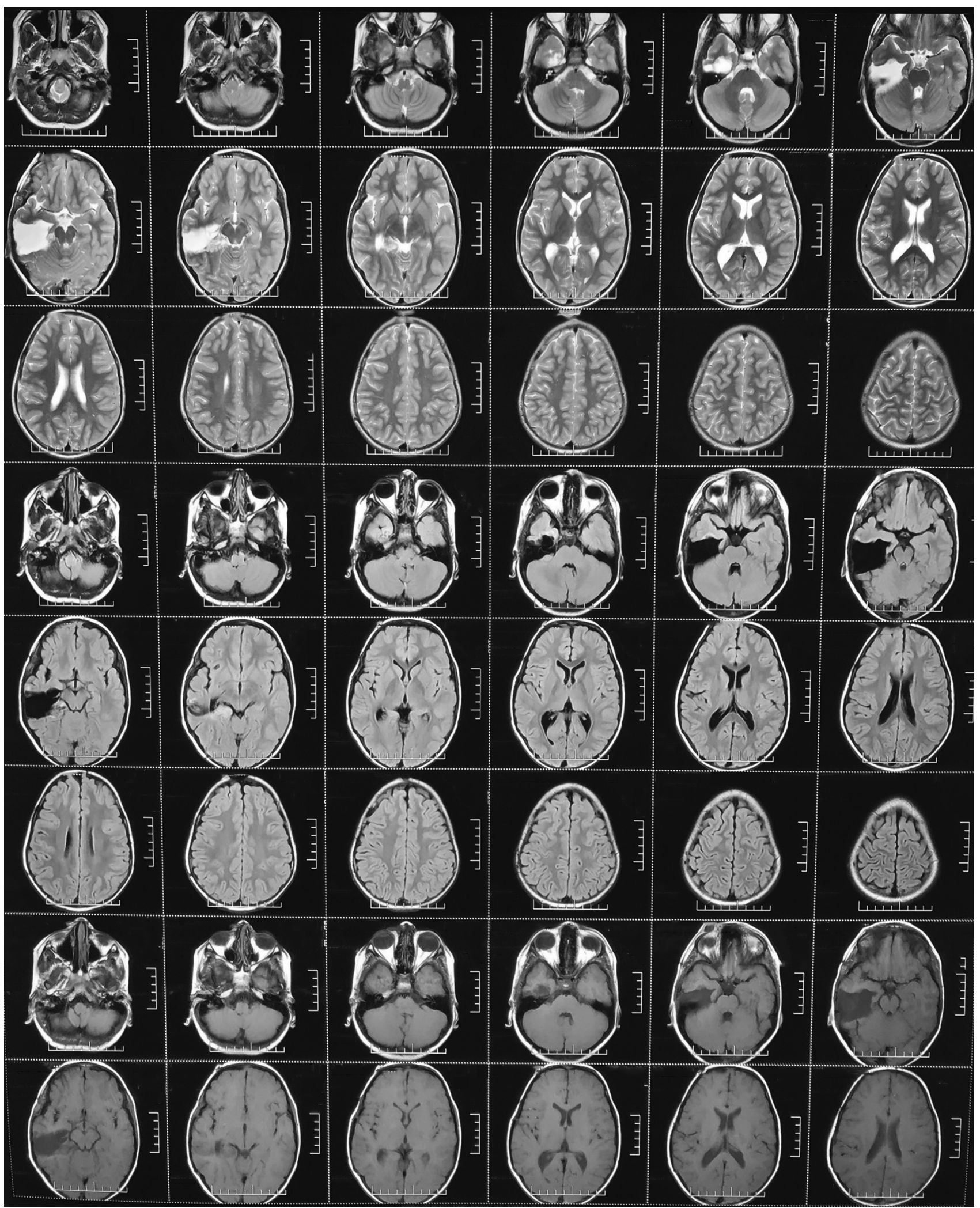

Fig. 5 Postoperative T2 magnetic resonance resonance imaging. 


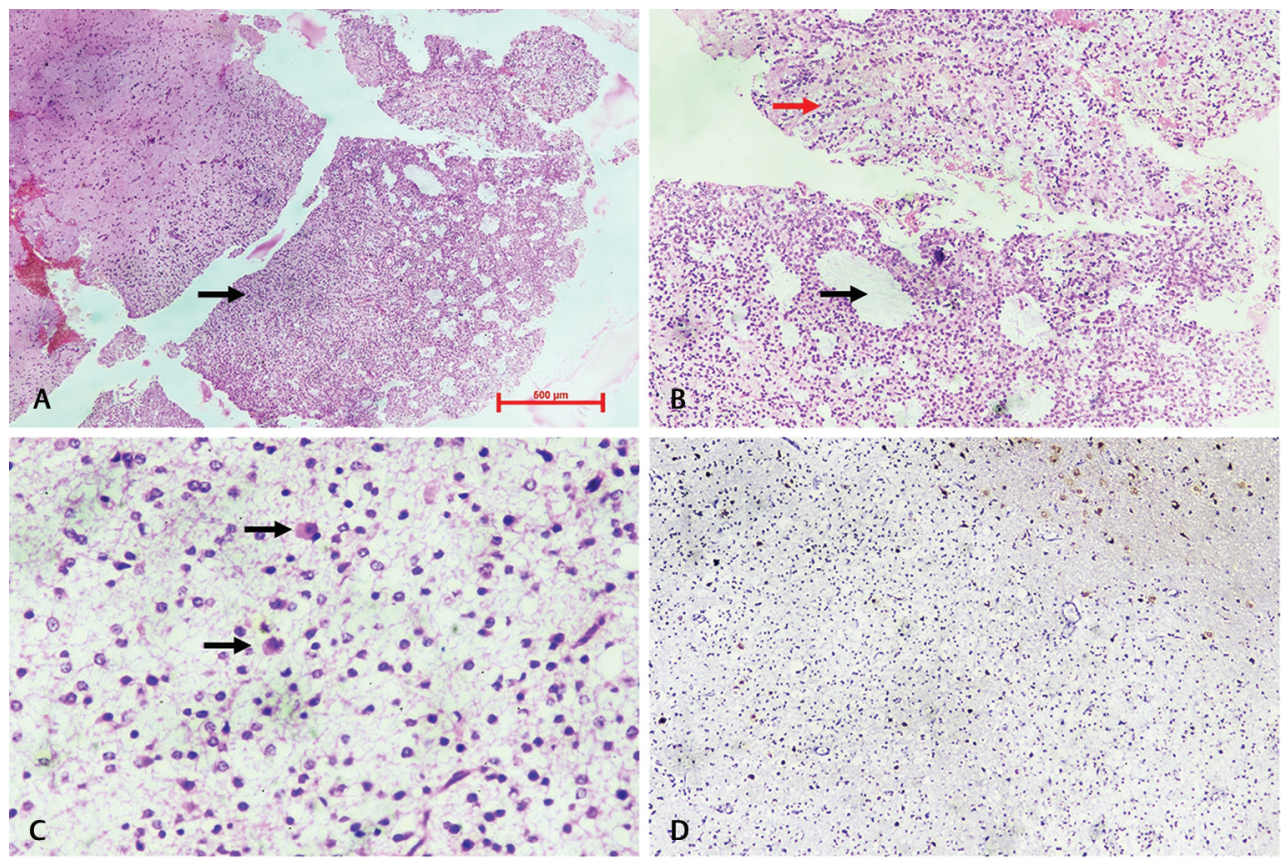

Fig. 6 Histopathology. (A) Representative images from tumor tissue (H\&E; 40x; arrow). (B) Microcytic areas (H\&E; 100x; black arrow) and specific glioneuronal elements (H\&E; 100x; red arrow). (C) Tumor containing oligodendrocyte-like cells in a mucoid background containing floating neurons (H\&E; 400x; arrows). (D) The neurons are immunopositive for NeuN(100x).

\section{Funding}

None.

\section{Conflict of interest}

None declared.

\section{References}

1 Daumas-Duport C, Scheithauer BW, Chodkiewicz JP. Laws ER Jr, Vedrenne C. Dysembryoplastic neuroepithelial tumor: a surgically curable tumor of young patients with intractable partial seizures. Report of thirty-nine cases. Neurosurgery 1988;23(5):545-556
2 Ranger A, Diosy D. Seizures in children with dysembryoplastic neuroepithelial tumours of the brain-A review of surgical outcomes across several studies. Childs Nerv Syst 2011;531:847-855

3 Raz E, Kapilamoorthy TR, Gupta AK, Fiorelli M. Case 186: dysembrioplastic neuroepithelial tumor. Radiology 2012; 265(1):317-320

4 Sukheeja D, Mehta J. Dysembryoplastic neuroepithelial tumor: a rare brain tumor not to be misdiagnosed. Asian J Neurosurg 2016;11(2):174 